\title{
EFECTO DEL MANEJO DE LAS MALEZAS EN MACADAMIA SOBRE LA POBLACIÓN DEL BARRENADOR DE LA NUEZ Ecdytolopha torticornis Meyrick (LEP: TORTRICIDAE) ${ }^{1}$
}

\author{
Helga Blanco-Metzler ${ }^{2}$, Rodolfo Villalobos $^{3}$, Allan D. Wat ${ }^{4}$, Derek Cosens ${ }^{5}$
}

\section{RESUMEN}

Efecto del manejo de las malezas en macadamia sobre la población del barrenador de la nuez Ecdytolopha torticornis Meyrick (Lep: Tortricidae). Entre junio y setiembre de 1992 se evaluó el efecto de tres sistemas de manejo de malezas; cobertura de cáscara de macadamia, chapia al ras del suelo y malezas creciendo libremente, sobre la población del barrenador de la nuez (Ecdytolopha torticornis) en plantaciones de macadamia en Turrialba, Costa Rica. El menor número de huevos del barrenador se registró en el tratamiento de suelo limpio, seguido por el tratamiento de malezas creciendo libremente y el de cobertura con cáscara. Aunque se ha utilizado este tipo de cobertura como una solución al problema de los desechos en el procesamiento de la nuez, el aumento de la población del barrenador bajo dicho tratamiento sugiere que podría estar actuando como atrayente del insecto adulto o bien confundiendo la población de parasitoides y depredadores. Es necesario probar otros sistemas de manejo de malezas con otros sus tratos, así como el uso de cultivos de cobertura entre las hileras de árboles, lo cual además podría incrementar las poblaciones de organismos benéficos.

\begin{abstract}
Effect of weed management on the population of macadamia nutborer Ecdytolopha torticornis Meyrick (LEP: Tortricidae). The effect of three weed management systems -macadamia husk as a mulch, bare ground and weeds growing freely - on the population ofthe nutborer, Ecdytolopha torticornis, were studied from June to September 1992 at Oriente Farm, Turrialba, Costa Rica. The smallest number of the nutborer's eggs was recorded on the bare ground treatment, followed by the unweeded treatment and the husk treatment. Although the macadamia husk as a mulch has been used to sol ve the problem of residues from nut processing, the results suggest that the use of this practice might increase the nutborer population by concentrating odours which could attract the adults, or by disrupting the parasitoid or predator population. There is a need for testing other mulches such as plastics, other plant residues, sawdust and others, and to study alternative weed management systems, including the use of cover crops in the mid-rows.
\end{abstract}

\section{INTRODUCCION}

El cultivo de la macadamia (Macadamia integrifolia) fue introducido a Costa Rica en 1952, pero no fue sino hasta 1965 cuando se inició su cultivo en forma comercial (González, 1978). Con el aumento en el área de siembra, se incrementaron también los problemas fitosanitarios del cultivo. Uno de estos lo constituye el barrenador de la nuez, Ecdytolopha torticornis (Meyrick) (Lep: Tortricidae), el cual, en su estado larval, ocasiona un daño directo al barrenar el interior de los frutos inmaduros y un daño indirecto al provocar la caída de fru- tos inmaduros (Blanco -Metzler, 1994; Blanco-Metzler, en preparación).

La cosecha de la macadamia se realiza recogiendo las nueces caídas bajo el dosel, por 10 que este debe estar libre de malezas en todo momento. Debido a que el sistema radical del árbol de macadamia es superficial, se deben encontrar alternativas al control químico o mecánico, ya que estos podrían dañar las raíces de los árboles.

El control de malezas mediante cultivos de cobertura o de materias inertes, es una práctica utilizada en

\footnotetext{
1 Centro de Investigación en Protección de Cultivos (CIPROC), Facultad de Agronomía, Universidad de Costa Rica.

2 Estación Experimental Fabio Baudrit, Universidad de Costa Rica. Apt. 183-4050 Alajuela. E-mail: hblanco@cariari.ucr.ac.cr

3 Centro Universitario del Atlántico, Universidad de Costa Rica

4 NERC, Institute of Terrestrial Ecology, Bush Estate, Penicuik, EH26 OQB, Midlothian, Scotland,UK

5 Institute ofCeH, Animal and Population Biology, The University ofEdinburgh, West Mains Road, Edinburgh EH9 3JT, Scotland, UK.
} 
muchos cultivos, tales como la palma africana, los huertos de manzana, las plantaciones de nueces y otros. El control de las malezas en plantaciones de piña en Hawaii, por ejemplo, se realiza por medio del uso de recubrimientos de papel (NAS, 1980), y en el establecimiento del cultivo de pejibaye (Bactris gasipaes) en el CA TIE, Costa Rica, se utilizan coberturas vivas de maní forrajero (Arachis pintoi) (Dominguez y de la Cruz, 1990). En macadamia, esta opción no se ha investigado en detalle, ni se conoce el efecto que diferentes coberturas puedan tener sobre la dinámica poblacional de plagas insectiles. Por lo tanto, se consideró importante investigar el efecto del manejo de las malezas en la abundancia de E. torticornis, mediante el uso de una cobertura de cáscara de macadamia, de la eliminación de las malezas con chapia al ras del suelo y el libre crecimiento de estas plantas.

\section{MATERIALES Y MÉTODOS}

La investigación se realizó entre junio y setiembre de 1992 en la Finca Oriente, Macadamia de Costa Rica, Turrialba, a una elevación de $650 \mathrm{msnm}$, una precipitación promedio de $2600 \mathrm{~mm}$ y una temperatura media de $22{ }^{\circ} \mathrm{C}$.

La plantación de la Finca Oriente tiene una edad promedio de 12 años, y comprende una mezcla de clones de macadamia (Macadamia integrifolia), entre los que destacan Keahou (Haes 246), Kau (Haes 344), Kakea (Haes 508) y Keaau (Haes 660) (Diego Pérez, comunicación personal).

Se estudiaron tres sistemas de manejo de malezas: cobertura de cáscara de macadamia, chapia al ras del suelo (suelo limpio) y malezas creciendo libremente, en tres sitios de la finca. La cáscara se tomó de depósitos de cáscara en descomposición. La cáscara de macadamia una vez recolectada de la planta procesadora no se puede aplicar directamente como cobertura, ya que durante la descomposición libera gran cantidad de calor. Debido a que las raíces de los árboles de macadamia se localizan en los primeron $30 \mathrm{~cm}$ del suelo, la adición de cáscara "fresca" podría provocar la quema de las raíces y afectar negativamente el crecimiento de los árboles. El tratamiento de suelo limpio se logró por medio de la chapia de las malezas a ras del suelo, cada tres semanas.

Los sitios fueron seleccionados con base a la edad de los árboles de macadamia (10 años), a la disponibilidad de los clones por sitio y al hábitat que los rodeaba. El sitio 1 presentaba una topografía plana, eestaba ubicado a la entrada de la finca Oriente, cerca de la calle principal y del río Pejibaye. Debido a la proximidad mucha de la cáscara proveniente de la planta procesa- dora era deposda en este lote El sitio 2 también presentaba una topografía plana, pero esttuado en las partes balas colinas, al río Pejibaye. Este si, a lo largo, presentaba árboles de Cassuasina sp. El sitio presentaba una topografía irregular, con $\mathrm{m}$ pendiente pronunciada, y estaba rodeado por árboles de Cassuasina, caña de azúcar, pastizales y macadamia intercalada con café.

Los tratamientos se establecieron un mes previo al inicio de los muestreos. Cada tratamiento comprendió al árbol de muestreo y los ocho árboles que los rodeaban con el fin de garantizar el efecto de los tratamientos sobre la población de E. torticornis. La distancia de siembra de los árboles de macadamia es de 6,5 metros, para un área de $169 \mathrm{~m}^{2}$ por grupo de árboles

El diseño experimental usado fue un factorial con tres sitios por tres tratamientos por cinco repeticiones. Se muestrearon 45 nueces ubicadas a una altura entre el nivel del suelo y 1,60 m, de acuerdo a las recomendaciones de Blanco-Metzler et al. (1996). Se evaluaron cinco repeticiones por tratamiento, para un total de 45 árboles. Las nueces fueron llevadas al laboratorio del Centro Agronómico Tropical de Investigación y Enseñanza, CA TIE $\left(23^{\circ} \mathrm{C}, 80 \% \mathrm{HR}\right)$, donde se registró el número de huevos del barrenador por nuez.

En este estudio no se registró la preferencia de oviposición para los diferentes clones, ya que blanco-Metzler (1994) encontró que no existen diferencias entre el número de huevos depositados en los diferentes clones.

Las malezas presentes en cada sitio fueron clasificadas como de hoja ancha o de hoja angosta.

Los efectos de los tratamientos se determinaron por medio de análisis de varianza, usando el programa SAS (SAS, 1985). Las diferencias entre medias se calcularon por medio de la prueba diferencia mínima significativa (DMS). Debido a que muchas nueces carecían de huevos, se transformó los datos mediante la fórmula $\sqrt{ } \mathrm{x}+0,5$.

\section{RESULTADOS}

Se inspeccionó un total de 9926 nueces. Se encontraron diferencias altamente significativas para el número de huevos por nuez para sitios $(\mathrm{F}=125,10 \mathrm{gl}=2$ $\mathrm{p}(0,001)$ y para tratamientos $(\mathrm{F}=9,71 \mathrm{gl}=2 \mathrm{P}(0,001)$. Sin embargo, la interacción sitio $\mathrm{x}$ tratamiento también fue altamente significativa $(\mathrm{F}=7,70 \mathrm{gl}=4, \mathrm{p}(0,001)$. El número de huevos fue menor en el sitio $2(0,21$ huevos por nuez), seguido por el sitio $1(0,40)$ y el sitio $3(0,48)$ donde se registró la mayor población del barrenador (Cuadro 1). 
Cuadro 1. Promedio de huevos de E. torticornis por nuez en tres sitios bajo tres sistemas de manejo de malezas en la Finca Oriente, Macadamia de Costa Rica, Turrialba.

\begin{tabular}{|c|c|c|c|c|c|}
\hline Sitio (1) & Media & Tratamiento (2) & Media & Sitio $x$ tratam.(3) & Media \\
\hline 3 & $0,48 a^{*}$ & Cáscara & $0,40 \mathrm{a}$ & 3-Malezas & $0,55 \mathrm{a}$ \\
\hline 1 & $0,40 \mathrm{~b}$ & Malezas & $0,35 \mathrm{~b}$ & 3-Cáscara & $0,50 a b$ \\
\hline \multirow[t]{7}{*}{2} & $0,21 \mathrm{c}$ & Suelo limpio & $0,32 \mathrm{bc}$ & 1- Cáscara & $0,47 \mathrm{abc}$ \\
\hline & & & & 3- Suelo limpio & $0,39 \mathrm{~cd}$ \\
\hline & & & & 1- Suelo limpio & $0,37 \mathrm{de}$ \\
\hline & & & & 1-Malezas & $0,36 \mathrm{def}$ \\
\hline & & & & 2- Cáscara & $0,24 \mathrm{~g}$ \\
\hline & & & & 2-Suelo limpio & $0,23 \mathrm{gh}$ \\
\hline & & & & 2-Malezas & $0,17 \mathrm{~h}$ \\
\hline
\end{tabular}

* Promedios seguidos por la misma letra no difieren significativamente según la Prueba de Diferencia Mínima Significativa $(\mathrm{P}<0,05)$.

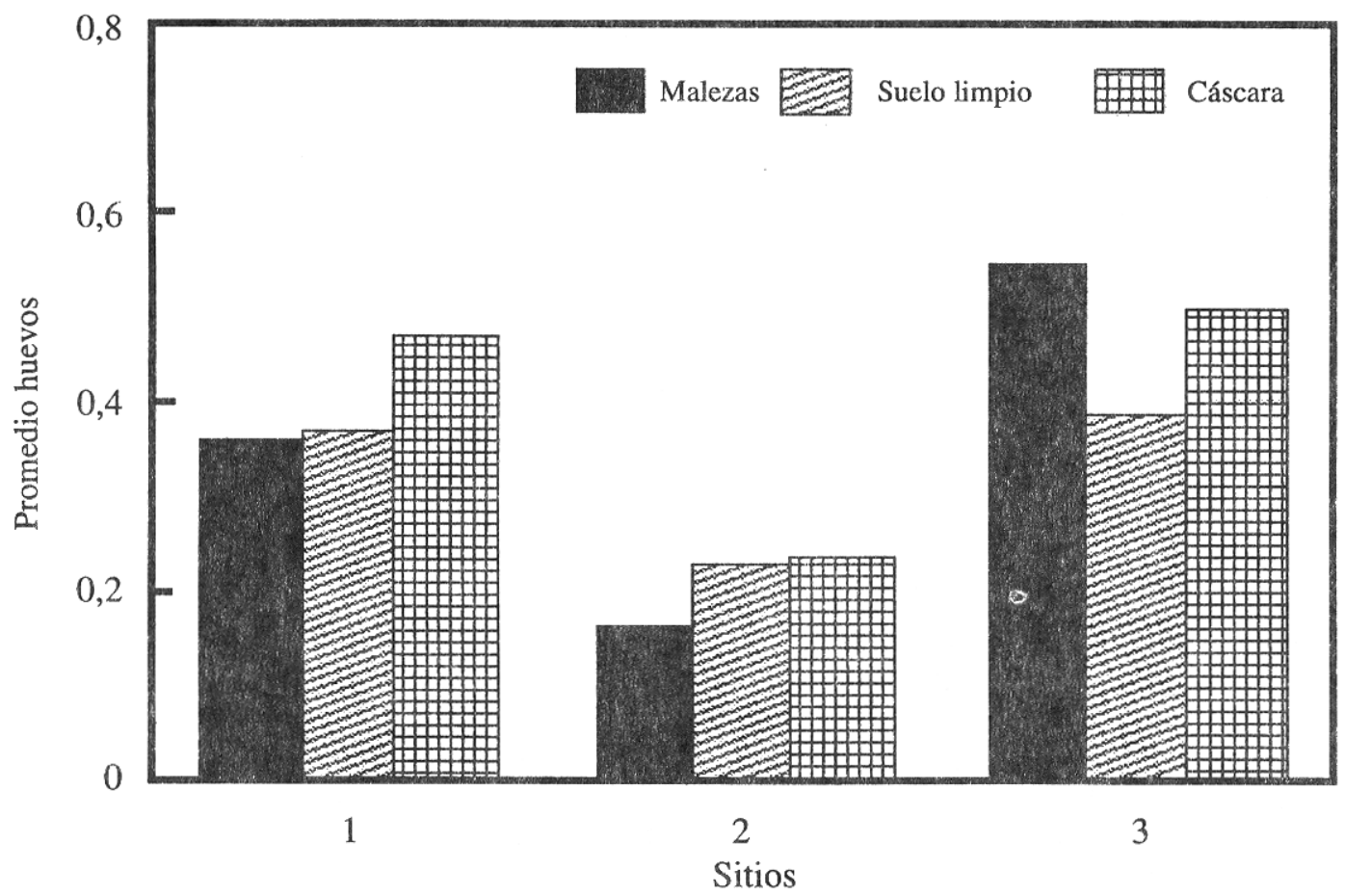

Fig. 1. Número promedio de huevos de E. torticornis por nuez en tres sitios y tres sistemas de manejo de malezas en la Finca Oriente, Turrialba, Costa Rica, 1992.

El menor número de huevos se registró con el suelo limpio $(0,32)$, seguido por el tratamiento de malezas creciendo libremente $(0,35)$ y la cobertura con cáscara $(0,40$ huevos por nuez) (Cuadro 1). La prueba DMS no pudo detectar diferencias entre el número promedio de huevos para los tratamientos con malezas y suelo limpio.
En el Cuadro 1 y Figura 1 se presenta el número promedio de huevos para la interacción sitio por tratamiento. Para los tres sitios, el tratamiento con cobertura presentó un mayor número de huevos que con el suelo limpio. El número de huevos para el tratamiento con malezas fue menor en los sitios uno y dos; sin embargo, la población más alta del barrenador se registró en el sitio tres. 
Las malezas de hoja ancha fueron: china (Impatiens balsamina), moriseco (Bidens pilosa), clavelillo (Emilia sonchifolia) y la escobilla (Sida rombifolia). Las gramíneas presentes fueron el pasto guinea (Panicum maximum), la pata de gallina (Eleusine indica), la caminadora (Rotboellia exaltata) y el arrocillo (Digitaria decumbens). Para los tres sitios, las malezas dominantes fueron las de hoja angosta, donde predominó el pasto guinea. Se encontraron diferencias en la composición de, malezas entre los sitios. En el sitio primero se presentó un número bajo de malezas de hoja ancha, donde ninguna de ellas dominaba, el sitio dos estaba dominado por $I$. balsamina, y en el sitio tres, casi no se encontraron malezas de hoja ancha. Este último sitio colindaba con un lote de caña de azúcar (Saccharum officinarum).

\section{DISCUSIÓN}

Las malezas son componentes naturales de un agroecosistema y juegan un papel importante en la diversidad y estabilidad de las poblaciones de insectos. La mayor parte de los estudios sobre malezas se concentran en el control químico o cultural, pero generalmene no se mencionan los beneficios que éstas producen (Altieri, et al., 1977). Entre las prácticas culturales para el manejo de malezas están el uso de plantas de cobertura, el uso de coberturas muertas como plásticos, residuos de plantas, aserrín y cascarilla de arroz, así como variaciones de laboreo del suelo (Ramiro de la Cruz, comunicación personal).

El número de huevos de E. torticornis difirió entre los sistemas de manejo de malezas empleados. Cuando se aplicó la cáscara de macadamia, se encontró un mayor número de huevos que en los sistemas con malezas o con suelo limpio, a excepción del sitio tres. La adición de cáscara podría afectar la población del barrenador al concentrar olores los cuales podrían atraer a los adultos, o confundiendo la población de parasitoides y depredadores. Altieri et al. (1977) informaron de una disminución en la colonización y reproducción de Epilachna varivestis en hábitats con malezas de hoja angosta y sugirió que éste era el resultado de unarepelencia o de un estímulo enmascarado de Eleusine indica y Leptochloa filiformis, dos malezas de hoja angosta comunes en el sitio.

La razón por la cual se registró el mayor número de huevos en el tratamiento con malezas en el sitio tres no es clara, sin embargo, podría haber un efecto por la colindancia de este sitio con la plantación de caña de azúcar. Una explicación probable por la cual se registró un menor número de huevos del barrenador en el sitio dos es que éste tenía una mayor abundancia de malezas de hoja ancha, lo cual, según Root (1973) y Andow (1990), aquellos hábitats con diversidad de vegetación, presentan un mayor número de parasitoides y depredadores. Según Roots (1973), la disminución en la abundancia de los insectos herbívoros en un hábitat diversificado se puede explicar por medio de las hipótesis sobre la concentración de los recursos y la hipótesis de los enemigos naturales.

La aplicación de cáscara de macadamiacomo método de control de malezas se presentó inicialmente como una alternativa importante, ya que esta práctica, además de solucionar el problema de las malezas, resolvía el problema de la finca sobre los residuos de la cáscara de la planta procesadora. Sin embargo, los resultados muestran un aumento en la población de $E$. torticornis por lo que se hace necesario probar otros sustratos. El uso de cultivos de cobertura entre las hileras de los árboles podría ser una alternativa para el manejo de las malezas y a la vez, aumentar las poblaciones de organismos benéficos. Otros beneficios que se le podrían atribuir al uso de coberturas vivas como las leguminosas, serían que ayudan a aumentar el contenido de nitrógeno del suelo, mejoran la estructura física del suelo, y disminuyen la erosión. Por otro lado, los cultivos de cobertura pueden competir con los árboles por agua y nutrimentos, interferir con la cosecha si estos no están bien manejados, y proveer refugio a ciertos insectos, los cuales, eventualmente podrían causar daño a la producción de macadamia (Bugg et al., 1991).

Hay una necesidad de probar estos sistemas de manejo de malezas por períodos más largos, y quizás, de ampliar el área de cada tratamiento. Por ejemplo, Altieri y Schmidt (1986) estudiaron el efecto de los cultivos de cobertura sobre la población de Cydia pomonella en una plantación de manzana. Durante el primer año de estudio, encontraron una leve disminución en el daño causado por esta plaga, pero al segundo año de establecido el sistema, el daño se redujo significativamente, de un 88 a un $4 \%$. Sus resultados sugieren que se requieren de mayores períodos para poder observar el efecto del manejo de malezas sobre las poblaciones de insectos. Además de los sistemas de manejo de malezas propuestos, sería recomendable estudiar otros sus tratos para el manejo de las malezas como el uso de plásticos, otros residuos vegetales, cascarilla de arroz y aserrín.

\section{LITERATURA CITADA}

ALTIERI, M.A.; VAN SCHOONHOVEN, A.; DOLL, J. 1977. The ecological role of weeds in insect pest management systems: a review illustrated by bean (Phaseolus vulgaris L.) cropping systems. PANS 23(2): 195205. 
ALTIERI, M.A.; SCHMIDT, L.L. 1986. Cover crops affect insect and spider populations in apple orchards. California Agriculture 40(1-2):15-17.

ANDOW, D.A. 1990. Population dynamics of an insect herbivore in simple and di verse habitats. Ecology 71(3): 1006-1017.

BLANCO-METZLER, H. 1994. The biology and ecology of the macadamia nutborer Ecdytolopha torticornis in Costa Rica. Tesis PhD. Universidad de Edimburgo/Instituto de Ecología Terrestre. 148 p.

BLANCO-METZLER,H.;WATT,A.D.;COSENS,D. 1996. Within-tree distribution of the macadamia nutborer Ecdyolopha torticornis Meyrick (Lep: Tortricidae). Journal of Economic Entomology (en prensa).

BUGG, R.L.; SARRANTONIO, M.; DUTCHER, J.D.; PHA T AK, S. C. 1991. U nderstory cover crops in pecan orchards: possible management systems. American Journal of Alternative Agriculture 6(2):50-62.

DE LA CRUZ, R 1988. Notas de clase, "Manejo integrado de malas hierbas". Turrialba, Costa Rica, CATIE. (Comunicación personal).
DOMINGUEZ, J.A.; DE LA CRUZ, R 1990. Competencia nutricional de Arachis pintoi Pinto como cultivo de cobertura durante el establecimiento de pejibaye Bactris gasipaes H.B.K. Manejo Integrado de Plagas (Costa Rica) 18:1-7.

GONZÁLEZ, O. 1978. La macadamia en Costa Rica. San José, Costa Rica, ICAFE. 4 p.

NATIONAL ACADEMY OF SCIENCES. 1980. Plantas nocivas y como combatirlas. México, Limusa. 574 p.

PÉREZ, D. 1990. Manejo de la plantación de macadamia. Atirro, Turrialba, Macadamia de Costa Rica. (Comunicación personal).

ROOT, RE. 1973. Organizationofaplant-arthropodassociation in simple and diverse habitats: the fauna collards. Ecological Monographs 43:95-124.

SAS INSTITUTE. 1985. SAS user's guide: statistics, version 5.1. Cary, North Carolina, SAS Institute. 